\title{
NUMERICAL SIMULATIONS OF ARTERIES WITH AN ADAPTIVE FINITE ELEMENT METHOD
}

\author{
A. Karolina Fuksa \\ Cracow University of Technology, Departament of Civil Engineering, Kraków, Poland \\ e-mail: afuksa@pk.edu.pl \\ WALDEMAR RACHOWICZ \\ Cracow University of Technology, Institute of Computer Science, Kraków, Poland \\ e-mail: wrachowicz@pk.edu.pl
}

\begin{abstract}
This paper presents applications of the adaptive FEM in computational biomechanics. The study involves finite deformation of artery walls built of layers of elastic, nearly incompressible (rubber-like) materials. The models are reinforced with nearly inextensible collagen fibres stretched along the directions close to the circumferential direction. A Total Lagrangian technique based on the displacement-pressure formulation is presented. Residual error estimates and finite element mesh adaptation strategies are developed. The procedures are illustrated for solutions of arteries under various load considerations.
\end{abstract}

Keywords: computational biomechanics, finite element method, adaptivity, error estimation, finite deformation

\section{Introduction}

Computational biomechanics is a rapidly developing branch of numerical simulations based on the Finite Element Method approximation. Research in the mechanics of soft tissues and particularly problems related to the simulations of aortic aneurysms and arteries with changes due to atherosclerosis are of particular interest. In both cases, the arteries are subjected to a high risk of rupture when pressurized. A commonly accepted approach for such biomechanical simulations is the use of low-order, very dense finite element (FE) meshes to discretize the biological objects of interest. Such an approach is motivated by the necessity for accurate approximation of a complex, patient-specific geometry involving a part of the artery. The main difficulties in obtaining a reliable simulation often lie in the ability to determine an appropriate constitutive relation between the stress and strain and also in the assessment of the material parameters through measurements. Representing accurately the mechanical behaviour of the real-life tissue is thus considered the main difficulty. The approximation error related to the discretization errors is seldom estimated or suppressed by successive mesh refinements, i.e. by convergence studies.

However, there exists a well established school of computational mechanics which showed that the discretization error can be controlled without loss of effciency (Demkowicz et al., 2007). One can use relatively coarse FE meshes and adapt them using $h$-refinements, i.e. changing locally the size of elements $h$, and/or $p$-refinements which involves changing locally the order of approximation $p$. The mesh adaptations are intended to reduce the estimated discretization error below a prescribed threshold for a minimum computational effort. The estimated error might be understood globally or it may concern some user-specified quantities of interest such as maximum equivalent stress. Such procedures are most often used for problems with simple and well-known physical models of the material. The question then arises whether the techniques of error estimation and mesh adaptivity could represent an advantage in computational biomechanics. Although the modeling error due to poor material description may be considered as the main cause of poor mechanical response predictions today, the situation may change so that the 
discretization error becomes important. Firstly, new effective versions of the finite element method arise such as the iso-geometric analysis (IGA) or the Discontinous Petrov-Galerkin (DPG) techniques which allow an accurate approximation of solutions on complex geometries using relatively coarse meshes. Secondly, the constant progress in the development of constitutive models and the recovery of their parameters may reduce in the future the dominance of this factor as a source of inaccuracy for simulations. Also, the interaction of biological tissues with medical devices such as stents may require error estimation. Finally, higher order approximation is in general more resistant to numerical locking which might be caused by the near-incompressibility and near-inextensibility of the arterial tissue reinforced with collagen fibres (Holzapfel and Gasser, 2001).

\section{Adaptive FEM for the mechanics of arteries}

In this study, we apply the methodology of error estimation and adaptivity for finite element simulations of arterial mechanics. Appropriate algorithms have already been developed and some adjustments have been made to obtain an accurate simulation. We developed the two-field formulation of displacement-pressure type (Simo and Taylor, 1991) with no inter-element continuity enforced for the pressure variable. The Total and Updated Lagrangian approaches can be used to solve the finite elasticity problem. We could also use an automatic $h$-adaptive algorithm (Demkowicz et al., 2007) to generate adaptive meshes based on the adjustment of the mesh to reduce the interpolation error of the solution. We developed an error-estimation technique which is applicable to finite elasticity problems. It is a version of the residual method proposed by Bank and Weiser (1985) in the context of linear elliptic boundary value problems. It may serve for error estimation of the solution in a global (energy) norm as well as errors of local quantities of interest in its goal-oriented version. The distribution of error indicators may also be used to guide adaptivity of finite element meshes. The adaptive FEM has been applied to solve typical problems in arterial mechanics.

A typical artery consists of concentric layers of tissue forming a tubular structure with properties significantly varying across the thickness. Such composites are nearly-incompressible and usually described as neo-Hookean materials reinforced with two families of collagen fibres. Atherosclerosis is a vascular disease which causes narrowing of the artery internal diameter and hardening of its walls as a result of plaque deposits. In some treatment therapies, a stent is inserted into the artery and is adequately deformed to widen the cross-section narrowed by atherosclerosis. A precise control of this treatment called angioplasty is necessary. It is a delicate procedure which requires sufficient widening of the artery without causing damage to the artery walls (Edelman and Rogers, 1998). In this research, we perform preliminary tests on a model of an artery under various load considerations for further investigations on the interaction between medical devices and biological tissues like for the case of angioplasty. Simulations of this sort involve a contact problem with large sliding and with finite deformation of the bodies in contact. The reliability of numerical simulations is also essential following the growth and remodelling (G\&R) of aneurysms. To start, we wish to assess the danger of rupture of an in vitro artery under various mechanical loads. Investigations of this kind involve modeling of a patient-specific geometry of the artery for which IGA is an interesting option. Such research becomes increasingly important for medical applications and the treatment of cardiovascular conditions.

\section{Rubber-like materials reinforced with fibres}

The tissue of a typical artery may be considered as a hyperelastic nearly-incompressible material reinforced with two families of collagen fibres (Holzapfel and Gasser, 2001) for which the stiffness grows exponentially with stretch in a way that they become nearly inextensible. The fibres are 
inactive if they are subject to compression. A common way to characterize a hyperelastic material consists of postulating its internal strain energy as a function of the invariants of the deformation. In the material description of the deformation of a body $\mathbf{x}=\mathbf{x}(\mathbf{X}, t), \mathbf{X}$ and $\mathbf{x}$ are the locations of a material particle in the initial and deformed configurations respectively, we define the right Cauchy-Green deformation tensor

$$
\mathbf{C}=\mathbf{F}^{\mathrm{T}} \mathbf{F}
$$

where $\mathbf{F}=\partial \mathbf{x} / \partial \mathbf{X}$ is the deformation gradient. Defining by $J=\operatorname{det}(\mathbf{C})^{1 / 2}=\operatorname{det}(\mathbf{F})>0$ the volume ratio, we can introduce the multiplicative decomposition of the deformation measures $\mathbf{F}$ and $\mathbf{C}$

$$
\mathbf{F}=J^{1 / 3} \overline{\mathbf{F}} \quad \mathbf{C}=J^{2 / 3} \overline{\mathbf{C}}
$$

where $\overline{\mathbf{F}}$ and $\overline{\mathbf{C}}$ are the unimodular deformation gradient and the Cauchy-Green tensor, respectively. We assume two families of fibre directions $\mathbf{a}_{i}(\mathbf{X}),|\mathbf{a}|=1, i=1,2$ which define the structural tensors

$$
\mathbf{A}_{i}=\mathbf{a}_{i} \otimes \mathbf{a}_{i} \quad i=1,2
$$

With the deformation measures defined above, we associate the following set of invariants

$$
I_{1}=\operatorname{tr}(\mathbf{C}) \quad I_{2}=\frac{1}{2}\left[(\operatorname{tr} \mathbf{C})^{2}-\operatorname{tr} \mathbf{C}^{2}\right] \quad I_{4 i}=\mathbf{C}: \mathbf{A}_{i} \quad i=1,2
$$

and their unimodular counterparts

$$
\bar{I}_{1}=\operatorname{tr}(\overline{\mathbf{C}}) \quad \bar{I}_{2}=\frac{1}{2}\left[(\operatorname{tr} \overline{\mathbf{C}})^{2}-\operatorname{tr} \overline{\mathbf{C}}^{2}\right] \quad \bar{I}_{4 i}=\overline{\mathbf{C}}: \mathbf{A}_{i} \quad i=1,2
$$

Further, we assume an additive split of the internal strain energy function into the volumetric, isochoric and fibre components

$$
W=W_{v o l}(J)+W_{i s o}\left(\bar{I}_{1}, \bar{I}_{2}\right)+\sum_{i=1}^{2} W_{f i}\left(\bar{I}_{4 i}\right)
$$

Splitting the internal energy into volumetric and isochoric parts for nearly incompressible isotropic materials is attributed to Flory (1961). The constitutive relation for the second PiolaKirchhoff stress tensor $\mathbf{S}$, which is implied by (3.5), reads

$$
\mathbf{S}=-p J \mathbf{C}^{-1}+2 J^{-2 / 3}\left\{W_{i s o, 1} \operatorname{Dev}[\mathbf{I}]-W_{i s o, 2} \operatorname{Dev}\left[\overline{\mathbf{C}}^{-2}\right]+\sum_{i=1}^{2} W_{f i, 4} \operatorname{Dev}\left[\mathbf{A}_{i}\right]\right\}
$$

where $p$ denotes the hydrostatic pressure

$$
p=-\frac{\partial W_{v o l}}{\partial J}
$$

and $\operatorname{Dev}[\bullet]=[\bullet]-1 / 3(\mathbf{C}:(\bullet)) \mathbf{C}^{-1}$. We assume the following form of the subsequent components of $W$ (Holzapfel and Gasser, 2001)

$$
W_{\text {vol }}=\frac{K}{4}(J-1)^{2} \quad W_{i s o}=\frac{\mu}{2}\left(\bar{I}_{1}-3\right) \quad W_{f i}=\frac{k_{1 j}}{2 k_{2 i}}\left\{\exp \left[k_{2 i}\left(\bar{I}_{4 i}-1\right)^{2}\right]-1\right\}
$$

where $K, \mu, k_{1 i}, k_{2 i}$ represent the parameters characterizing the material. 


\section{Formulation of the problem and finite element approximation}

Typically, the parameter $K$ is significantly larger than $\mu$, reflecting the fact that the material is nearly incompressible. It becomes convenient to formulate a two-field mixed problem with displacements, $\mathbf{u}=\mathbf{x}-\mathbf{X}$, and the pressure $p$ as the unknowns. This involves the principle of virtual work and the weak form of the constitutive formula for the pressure (Simo and Taylor, 1991)

$$
\begin{array}{ll}
\int_{\Omega} \mathbf{S}: \frac{1}{2}\left(\mathbf{F}^{\mathrm{T}} \boldsymbol{\nabla}_{X} \mathbf{v}+\nabla_{X}^{\mathrm{T}} \mathbf{v F}\right) d X=\int_{\Omega} J \mathbf{b} \cdot \mathbf{v} d X+\int_{\Gamma^{N}}\left(\mathbf{F}^{\mathrm{T}} \mathbf{v}\right) \cdot \widehat{\mathbf{t}} d A & \forall \mathbf{v} \in V \\
\int_{\Omega}-\left(\frac{2}{K} p-(J-1)\right) q d X=0 & \forall q \in L^{2}(\Omega)
\end{array}
$$

In this statement, $\mathbf{b}$ denotes the forces per unit volume, $\hat{\mathbf{t}}$ are the tractions prescribed on the Neumann part of the boundary $\Gamma^{N}, \mathbf{v}$ and $q$ are appropriate test functions with $V=\left\{\mathbf{v} \in H^{1}(\Omega): \mathbf{v}=0\right.$ on $\left.\Gamma^{D}\right\}$ and $\Gamma^{D}$ stands for the part of $\partial \Omega$ with Dirichlet boundary conditions. This nonlinear problem can be linearized and solved with the Newton-Raphson procedure. The linearized problem takes the following form

$$
\begin{array}{ll}
a(\Delta \mathbf{u}, \mathbf{v})+b(\Delta p, \mathbf{v})=l_{1}(\mathbf{v}) & \forall \mathbf{v} \in H^{1}(\Omega) \\
b(q, \Delta \mathbf{u})-c(\Delta p, q)=l_{2}(q) & \forall q \in L^{2}(\Omega)
\end{array}
$$

where the bilinear forms are defined as follows

$$
\begin{aligned}
& a(\Delta \mathbf{u}, \mathbf{v})=\int_{\Omega} \mathbf{E}^{\prime}(\mathbf{u}, \mathbf{v}):\left[C_{d e v}-p J\left(\mathbf{C}^{-1} \otimes \mathbf{C}^{-1}-2 \mathbf{C}^{-1} \odot \mathbf{C}^{-1}\right)\right]: \mathbf{E}^{\prime} \\
& \quad+\boldsymbol{\nabla}_{X} \mathbf{v}:\left[\boldsymbol{\nabla}_{X} \Delta \mathbf{u}\left(\operatorname{Dev}[\mathbf{S}]-p J \mathbf{C}^{-1}\right)\right] d X \\
& b(\Delta p, \mathbf{v})=-\int_{\Omega} J \nabla_{X} \cdot \mathbf{v} \Delta p d X
\end{aligned}
$$

where

$$
\boldsymbol{\nabla}_{X} \cdot \mathbf{v}=\mathbf{C}^{-1}: \mathbf{E}^{\prime}(\mathbf{u}, \mathbf{v}) \quad \mathbf{E}^{\prime}(\mathbf{u}, \mathbf{v})=\frac{1}{2}\left(\nabla_{X}^{\mathrm{T}} \mathbf{v} \mathbf{F}+\mathbf{F}^{\mathrm{T}} \boldsymbol{\nabla}_{X} \mathbf{v}\right)
$$

and

$$
C_{d e v}=2 \frac{\partial}{\partial \mathbf{C}}\left\{J^{-2 / 3} \operatorname{Dev}\left[2 \frac{\partial W}{\partial \mathbf{C}}\right]\right\}
$$

denotes the tensor of elasticities. The linear functionals $l_{i}(\mathbf{v})$ correspond to the residuum of the current approximation $(\mathbf{u}, p)$. To discretize the problem hexahedral elements of the $Q^{p} / P^{p-1}$ family are used where $p$ is the order of approximation. It provides a stable approximation of the mixed problem.

\section{Error estimation and adaptivity}

Estimation of the approximation error of the mixed formulation of finite elasticity problem (4.1) was considered by Rüter and Stein (2000). It is based on the Helmholtz decomposition of the error in the first Piola-Kirchhoff stress tensor, $\mathbf{P}-\mathbf{P}_{h}=\boldsymbol{\nabla} \boldsymbol{\psi}+\boldsymbol{\nabla} \times \boldsymbol{\phi}$, where $\boldsymbol{\psi}$ and $\phi$ are the 
error functions. Following this representation, the residual of equilibrium equation $(4.1)_{1}$ can be written as follows

$$
\begin{aligned}
R(\mathbf{v}) & =\int_{\Omega} J \mathbf{b} \cdot \mathbf{v} d X+\int_{\Gamma^{N}} \hat{\mathbf{t}} \cdot\left(\mathbf{F}^{\mathrm{T}} \mathbf{v}\right) d A-\int_{\Omega} \mathbf{P}_{h}: \nabla \mathbf{v} d X \\
& =\int_{\Omega}\left(\mathbf{P}-\mathbf{P}_{h}\right): \nabla \mathbf{v} d X=(\boldsymbol{\psi}, \mathbf{v})_{1}
\end{aligned}
$$

which defines the function $\psi$ as a solution to the variational boundary-value problem

$$
\text { Find } \boldsymbol{\psi}:(\boldsymbol{\psi}, \mathbf{v})_{1}=R(\mathbf{v}) \quad \forall \mathbf{v} \in V
$$

The norm of $\boldsymbol{\psi}$ is estimated using the technique of error estimation with self-equilibrated element residuals due to Ainsworth and Oden (1992). The final estimate of the error in displacements and in pressure is found as follows

$$
\left|\mathbf{u}-\mathbf{u}_{h}\right|_{1, \Omega}+\left\|p-p_{h}\right\|_{0, \Omega} \leqslant C\left(|\psi|_{1, \Omega}+\left\|U-\frac{2}{K} p_{h}\right\|_{0, \Omega}\right)
$$

where $U=J-1$. That is, it includes the norm of the error function $\psi$ and the measure of the dissatisfaction of the constitutive relation for pressure.

We modify this approach by replacing the method of self-equilibrated residuals by the element residual technique proposed by Bank and Weiser (1985). In this technique, we assume that $V_{h p}(K)$ is the space of shape functions of the element $K$. We consider an enriched version of this space $V_{h, p+1}(K)$, which is obtained by enriching the order of approximation by one. Let $\Pi_{h p}$ be an interpolation operator defined for $V_{h p}(K)$, for instance of the Lagrangian type. We consider a kernel of this operator in $V_{h, p+1}(K)$

$$
M_{K}=\left\{\mathbf{v} \in V_{h, p+1}: \Pi_{h p} \mathbf{v}=0\right\}
$$

For every element $K$, we solve a discrete local boundary-value problem for the error indicator function $\phi_{K}$

$$
\phi_{K} \in M_{K}: \quad a\left(\phi_{K}, \mathbf{v}\right)=R_{K}(\mathbf{v}) \quad \forall \mathbf{v} \in M_{K}
$$

where $R_{K}$ are the element residuals and $a(\cdot, \cdot)$ is a bilinear form of the problem. The estimate of the dual norm of the global residual is expressed as follows

$$
\|R\|_{*} \leqslant C\left(\sum_{K}\left\|\phi_{K}\right\|_{E, K}^{2}\right)^{1 / 2}
$$

where $\|\cdot\|_{E}$ is the energy norm associated with the form $a$. An advantage of this technique is that it is reliable and inexpensive as the size of the local problems is $O\left(p^{2}\right)$ instead of $O\left(p^{3}\right)$ for other techniques including the method of self-equilibrated residuals.

The distribution of error indicators is a basis for local refinements of the finite element mesh. A standard feedback mesh adaptation algorithm divides the elements with the largest errors and finds the solution on the new mesh, and so on. It can be stated as follows:

1. Solve the problem on the current mesh.

2. Find the error indicators $\eta_{K}$.

3. Stop if $\left(\sum_{K} \eta_{K}^{2}\right)^{1 / 2} \leqslant T O L$.

4. Refine the elements $\mathrm{K}$ for which: $\eta_{K}>\alpha \max _{L} \eta_{L}$.

5. Go to 1 .

Here, $0<\alpha<1$ is a user-defined parameter controlling the adaptive process. 


\section{Numerical examples}

\subsection{Yosibash and Priel benchmark}

In the first example, we consider the effect of pressurizing a typical artery such as the left anterior descending coronary artery (LAD) which consists of two main layers, the internal media and the external adventitia layer. This problem was investigated as a benchmark test by Yosibash and Priel (2011). The geometry of the artery can be modeled as a cylindrical tube composed of two layers of the material. The material data for the media is $K=100000 \mathrm{kPa}, \mu=54.0 \mathrm{kPa}$, $k_{1}=0.64 \mathrm{kPa}$ and $k_{2}=3.54$ and the fibres are inclined with respect to the horizontal direction at an angle of $\pm 10^{\circ}$. In the adventitia layer, $K=100000 \mathrm{kPa}, \mu=5.4 \mathrm{kPa}, k_{1}=5.1 \mathrm{kPa}$ and $k_{2}=15.4$ and the fibres are inclined with respect to the horizontal direction at an angle of $\pm 40^{\circ}$. The artery is pressurized by an internal pressure of $13.3 \mathrm{kPa}(100 \mathrm{~mm} \mathrm{Hg})$. Tetrahedral elements with the $Q^{2} / P^{1}$ shape functions are used. Figure 1 shows the geometry of the artery, the deformed configuration as well as the pressure distribution.
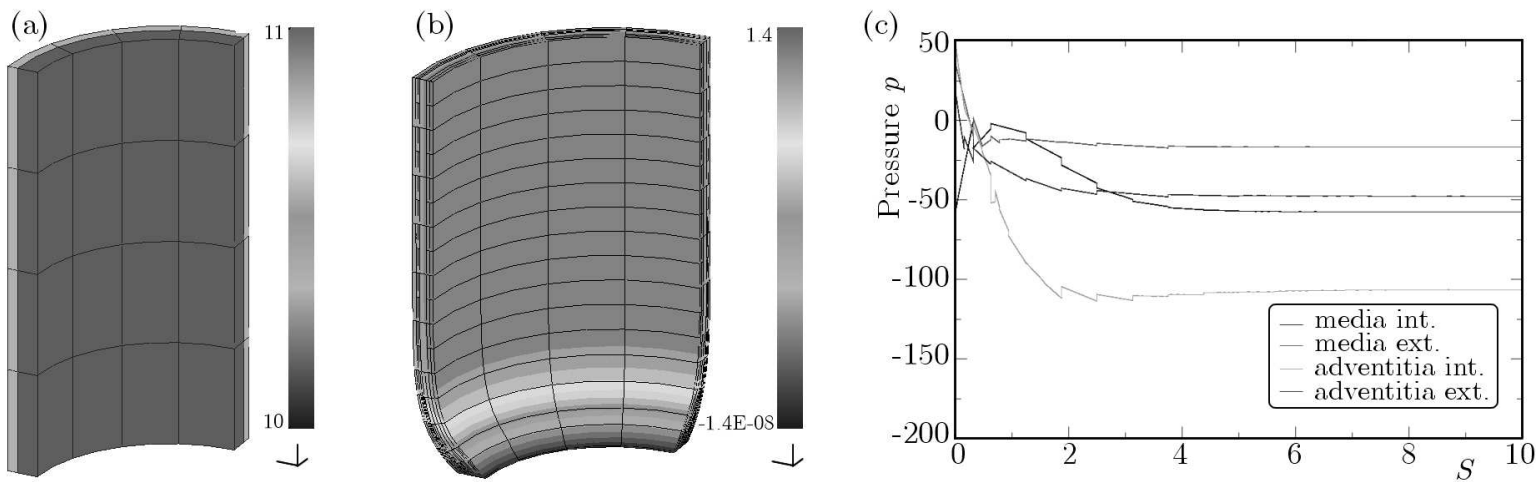

Fig. 1. Yosibash and Priel benchmark: (a) classes of the anisotropic material, (b) radial displacements,

(c) pressure along the layers of media and adventitia

\subsection{LAD aneurysm}

In the following test, a model of the artery similar to the one used in the Yosibash and Priel benchmark is considered. However, an additional cavity within the media layer is created to model the medical condition of aneurysm. The material data remains the same as in the previous example and the artery is subjected to an internal pressure of $13.3 \mathrm{kPa}$. The distribution of the deviatoric stress and pressure on the surface of the artery are shown in Fig. 2.

\subsection{Carotid bifurcation}

A typical geometry of an artery such as a carotid bifurcation is depicted in Fig. 3. Also shown are the classes of the material, the media and the adventitia layers for which the material parameters are $K=100000 \mathrm{kPa}, \mu=35.74 \mathrm{kPa}, k_{1}=13.9 \mathrm{kPa}, k_{2}=13.2$. The fibres are inclined with respect to the circumferential direction at an angle of $\pm 17.8^{\circ}$ in the media layer and an angle of $\pm 30.1^{\circ}$ in the adventitia layer. In addition, the directions of the fibres are orthogonal in the area of bifurcation and parallel in the patch located in the vicinity of the saddle point. The artery is pressurized with an internal pressure of $5.5 \mathrm{kPa}$. Figures $3 \mathrm{~b}$ and $3 \mathrm{c}$ show the pressure distribution over the external and internal surface of the artery model. The distribution of the deviatoric stress over the external surface is shown in Fig. 4. 


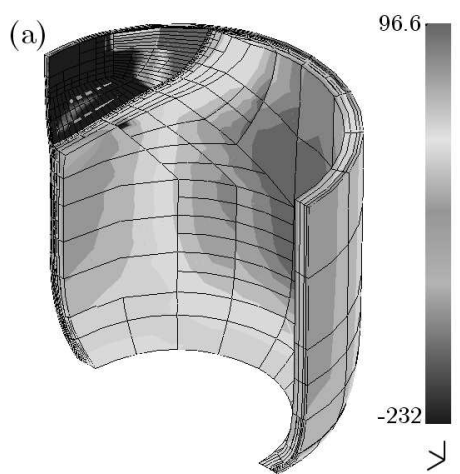

(b)

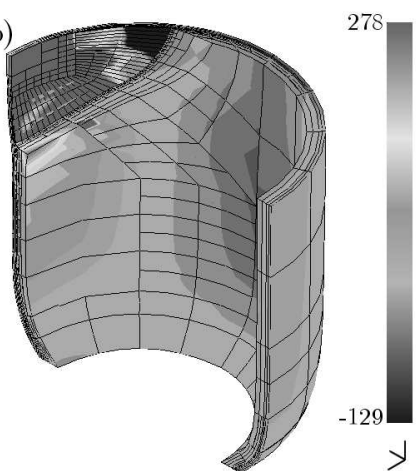

(c)

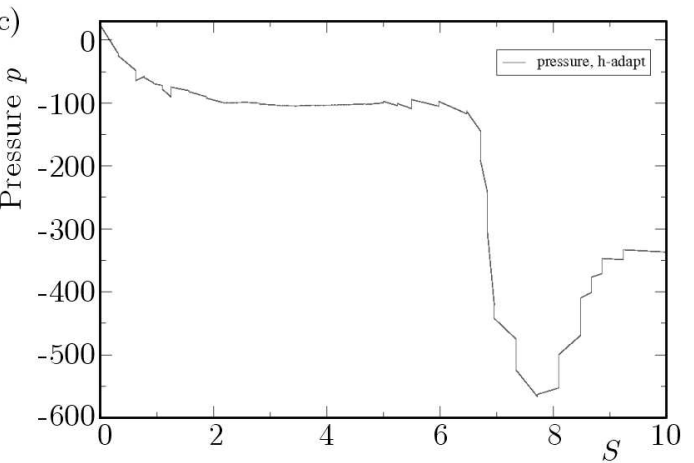

Fig. 2. LAD aneurysm: (a) $\operatorname{Dev}[\mathbf{S}]_{x x}$, (b) $\operatorname{Dev}[\mathbf{S}]_{y y}$, (c) pressure along the external surface of the adventitia

(a)

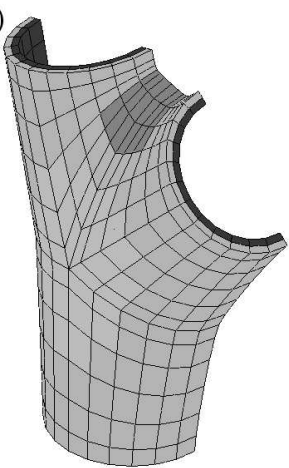

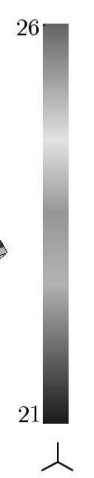

(b)

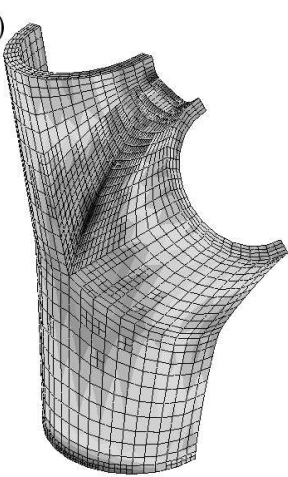

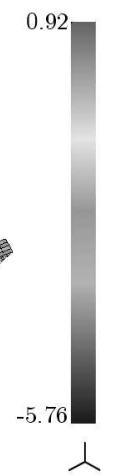

(c)

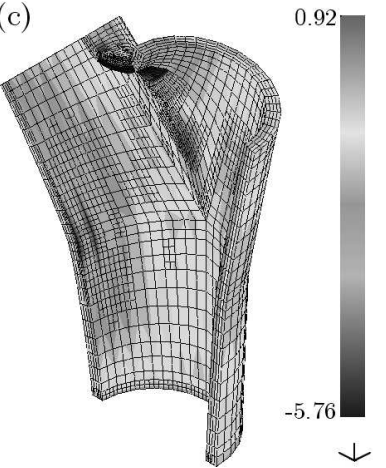

Fig. 3. A carotid bifurcation under internal pressure: (a) classes of the anisotropic material, (b) distribution of pressure on the external and (c) internal surface of the artery
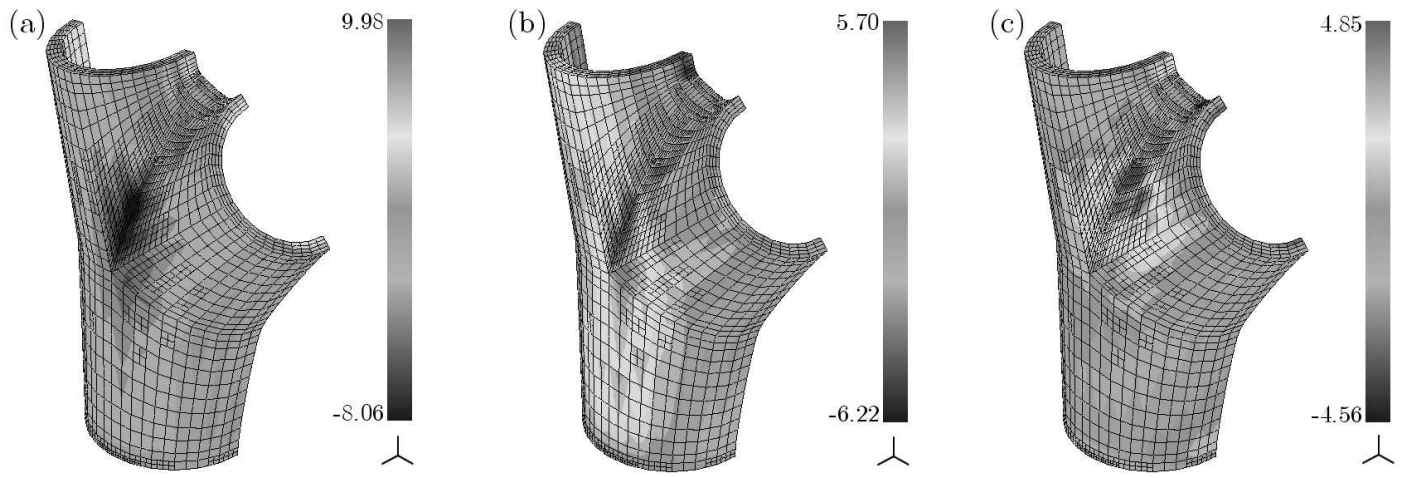

Fig. 4. A carotid bifurcation under internal pressure: (a) $\operatorname{Dev}[\mathbf{S}]_{x x}$, (b) $\operatorname{Dev}[\mathbf{S}]_{y y}$, (c) $\operatorname{Dev}[\mathbf{S}]_{z z}$ 


\subsection{Contact with a rigid body}

The future goal of this research is the investigation of the interaction between an artery and a medical device. It involves a contact problem of deforming bodies. In this Section, we present a preliminary test of contact with a rigid obstacle.

In this problem, the contact of an elastic cylinder of radius $r=8 \mathrm{~cm}$ and a rigid obstacle such as a cylinder of radius $r=20 \mathrm{~cm}$ is considered. The material parameters of the cylinder are $K=100000 \mathrm{kPa}, \mu=1.0 \mathrm{kPa}$ without fibres in this case. Only normal contact is considered. The normal gap function is defined as follows. Given a point of the elastic cylinder located at $\mathbf{x}^{2}$ we find its closest point $\overline{\mathbf{x}}^{1}$ on the obstacle and the unit outward normal on this surface $\overline{\mathbf{n}}^{1}$. The gap function is set as

$$
g_{N}=\left(\mathbf{x}^{2}-\overline{\mathbf{x}}^{1}\right) \cdot \overline{\mathbf{n}}^{1}
$$

No penetration of the bodies is expressed as $g_{N} \geqslant 0$, with an additional condition that the normal stress on the contact area is negative if $g_{N}=0$, while for $g_{N}>0$ it vanishes. We enforce these conditions by adding to the load side of formulation $(4.1)_{1}$ the penalty term

$$
\int_{\Gamma_{c}} \frac{1}{\epsilon} g_{N} \delta g_{N} d A
$$

where $\epsilon$ is a small penalty parameter and $\Gamma_{c}$ is the contact area. The penalty term is activated only if $g_{N} \leqslant 0$. The variation $\delta g_{N}=\mathbf{v} \cdot \overline{\mathbf{n}}^{1}$ where $\mathbf{v}$ stands for a variation of displacements (a test function). Appropriate linearization of the penalty term is necessary in order to solve the nonlinear problem of finite elasticity with contact with the Newton-Raphson iteration (Wriggers, 2002). Selected characteristics of the solution are presented in Fig. 5. A similar problem of contact of an elastic sphere with a horizontal rigid plane is illustrated in Fig. 6.
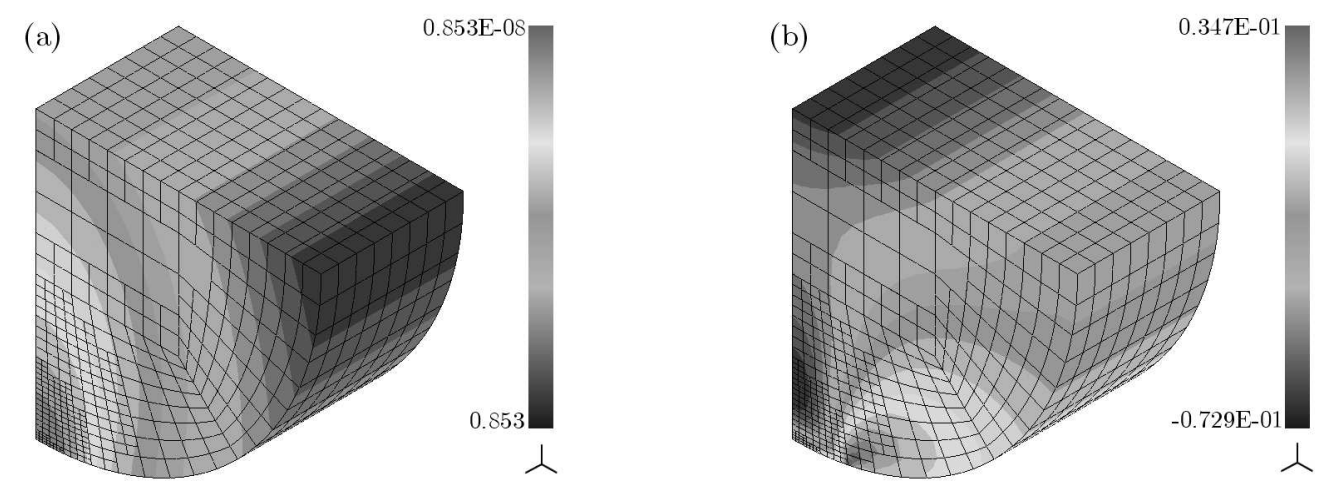

Fig. 5. Contact of the cylinder and a rigid obstacle: (a) displacements $u_{z}$, (b) $\operatorname{Dev}[\mathbf{S}]_{z z}$
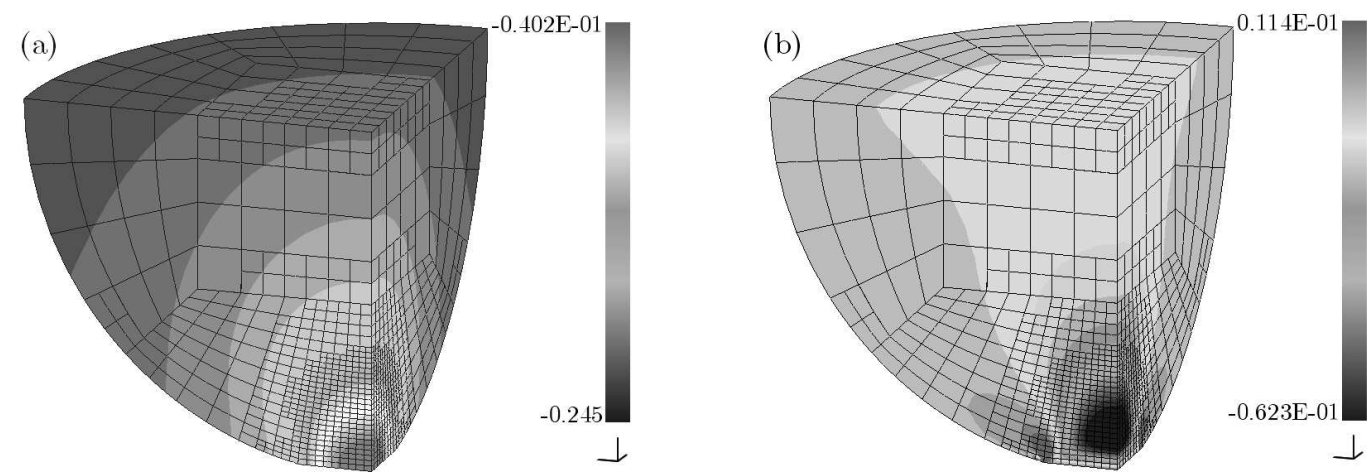

Fig. 6. Contact of an elastic sphere with a rigid plane: (a) displacements $u_{z}$, (b) Dev $[\mathbf{S}]_{z z}$ 


\section{Conclusions}

This study investigates the use of the adaptive version of the FEM in the biomechanics of arteries. In particular, the adaptive FEM has been applied to simulate the behaviour of a typical artery subjected to internal pressure, the pressurization of an artery with aneurysm as well as a contact problem. Such simulations serve as preliminary studies for further research on the interaction between a medical device and biological tissues. The material of the artery has been modeled as nearly-incompressible, neo-Hookean composite reinforced with two families of collagen fibres. The Total Lagrangian technique based on the displacement-pressure formulation has been presented as well as residual error estimate and finite element mesh adaptation strategies. Finally, an adaptive FEM has been applied to solve typical problems in arterial biomechanics.

Acknowledgement

This work was supported by the Polish National Science Centre under grant number UMO2011/01/B/ST6/07306

\section{References}

1. Ainsworth M., Oden J.T., 1992, A procedure for a posteriori error estimation for h-p finite element methods, Computer Methods in Applied Mechanics and Engineering, 101, 73-96

2. Bank R.E., Weiser A., 1985, Some a posteriori error estimates for elliptic partial differential equations, Mathematics of Computation, 44, 283-301

3. Demkowicz L., Kurtz J., Pardo D., Paszyński M., Rachowicz W., Zdunek Z., 2007, Computing with hp Finite Elements. II. Frontiers: Three-Dimensional Elliptic and Maxwell Problems with Applications, CRC Press, Taylor and Francis

4. Edelman E.R., Rogers C.R., 1998, Pathobiologic responses to stenting, American Journal of Cardiology, 81, 4E-6E

5. FLORY R., 1961, Thermodynamic relations for highly elastic materials, Transactions of the Faraday Society, 57, 829-838

6. Holzapfel G.A., Gasser Th.C., 2001, A viscoelastic model for fibre-reinforced composites at finite strains: Continuum basis, computational aspects and applications, Computer Methods in Applied Mechanics and Engineering, 190, 4379-4403

7. RÜter M., Stein E., 2000, Analysis, finite element computation and error estimation in transversely isotropic nearly incompressible finite elasticity, Computer Methods in Applied Mechanics and Engineering, 190, 519-541

8. Simo J.C., TAYlor R.L., 1991, Quasi-incompressible finite elasticity in principal stretches, Computer Methods in Applied Mechanics and Engineering, 85, 273-310

9. Wriggers P., 2002, Computational Contact Mechanics, John Wiley \& Sons, 520 p.

10. Yosibash Z., Priel E., 2011, p-FEMs for hyperelasic nearly-incompressible materials under finite deformations with applications to arteries simulation, International Journal for Numerical Methods in Engineering, 88, 1152-1174 